\title{
Aerobic bacteriology of Chronic Suppurative Otitis Media (CSOM) in a tertiary care hospital: A retrospective study
}

\author{
R. K. Sanjana ${ }^{1}$, Y. I. Singh ${ }^{2}$, N.S.Reddy ${ }^{3}$ \\ ${ }^{1}$ Assistant Professor, ${ }^{2}$ Prof \& Head, Dept of Microbiology, ${ }^{3}$ Prof \& Head, Dept. of Ear Nose and Throat (ENT), College of \\ Medical Sciences- Teaching Hospital, Bharatpur, Chitwan District, Nepal.
}

\begin{abstract}
The present study was carried out to determine the aerobic microorganisms involved and their antibiotic sensitivity pattern in patients with Chronic Suppurative Otitis Media (CSOM) and to provide a guideline for empirical antibiotic therapy.
\end{abstract}

Between March 2009 to February 2011, the ear discharge samples submitted at the microbiology laboratory were processed aerobically and all the isolates were included in this study. All organisms were identified morphologically and biochemically by standard laboratory procedures and antibiotic susceptibility pattern was determined by modified Kirby Bauer disc diffusion method as per National Committee for Clinical Laboratory Standard recommendations.

Out of a total of 214 samples, microbiological culture was yielded from 202 (94.3\%) specimens. Single organism were isolated from $168(78.5 \%)$ of the culture positive specimens, while the remaining 34 (15.9\%) had two or more organisms isolated. Pseudomonas aeruginosa 59 (35.1\%) was the most common isolate, followed by Staphylococcus aureus 42 (25.0\%) including 5 (3\%) of the Methicillin Resistant Staphylococcus Aureus (MRSA). Antibiotic sensitivities of Pseudomonas aeruginosa showed that $93.2 \%$ isolates were sensitive to tobramycin, whereas, $91.5 \%$ isolates were sensitive to ceftazidime and $77.9 \%$ to amikacin.Only $50.8 \%$ of the isolates of Pseudomonas aeruginosa were sensitive to ciprofloxacin and $25.4 \%$ to gentamicin. For Staphylococcus aureus (other than MRSA) 95.2\% were sensitive to cloxacillin, $83.3 \%$ to gentamicin and $78.5 \%$ to erythromycin. Only $07.1 \%$ were sensitive to ampicillin and $26.1 \%$ to ciprofloxacin.

Pseudomonas aeruginosa was the most common isolate followed by Staphylococcus aureus. More than 90\% of Pseudomonas and 90\% of Staph aureus were sensitive to tobramycin and cloxacillin respectively. Therefore, these two drugs may be included in the formulary to cover the most common aerobic isolates involved in CSOM.

Key words: Chronic suppurative otitis media, Pseudomonas aeruginosa, antibiotic sensitivity.

Correspondence: R.K. Sanjana

E-mail: rajkumari_sanjana@yahoo.co.in 


\section{Introduction}

Chronic suppurative otitis media (CSOM) is a commonly encountered infection of the middle ear. The most acceptable definition is that CSOM is an infection of the middle ear that lasts more than 3 months and is accompanied by tympanic membrane perforation. ${ }^{1}$ It is one of the most common diseases of all age groups, especially of childhood. The disease is prevalent in developing countries and is a disease of the poverty. ${ }^{2}$

Chronic suppurative otitis media has profound impact on society in terms of resources utilized in treatment and direct impact that chronic infection has on hearing on patient. ${ }^{3}$ It causes conductive and sensorineural hearing loss and adverse effect on childhood development. ${ }^{4}$ The first line of treatment of CSOM is topical therapy. Concerns about ototoxocity leads to decline in use of aminoglycosides containing ear drops.Quinolone containing ear drops exhibit excellent antipseudomonal and broad spectrum antimicrobial activity and non-ototoxicity. ${ }^{5,6}$

In chronic suppurative otitis media the most frequently isolated bacteria are Pseudomonas aeruginosa, Staphylococcus aureus, Proteus species and Klebsiella species. ${ }^{2}$ Knowledge of the local pattern of infection is essential to enable efficacious treatment of this disorder.

This study is aimed at finding the local pattern of aerobic microbes involved and their antimicrobial sensitivity pattern in cases of chronic suppurative otitis media to provide a guideline for empirical antibiotic therapy.

\section{Materials and methods}

This study was based on retrospective data of samples sent from the department of ear, nose and throat (ENT) of College of Medical SciencesTeaching Hospital, Bharatpur. A total of 214 purulent discharge samples from the clinically diagnosed cases of CSOM during March 2009 to February 2011 that were sent for culture and sensitivity to the Microbiology lab were included in this study. Sterile cotton swab sticks were used to collect the samples.

The pus swabs were cultured on Blood and MacConkeys agar and incubated aerobically at $37^{\circ} \mathrm{C}$ for an overnight. All organisms isolated were identified according to standard microbiological methods. ${ }^{7}$ Antimicrobial susceptibility test was performed using Kirby- Bauer disc diffusion method and updated by Clinical and Laboratory Standards Institute guidelines. ${ }^{8}$ The standard antimicrobial discs (HI Media Laboratoriers, Pvt. Ltd, Mumbai) used for Staph aureus were oxacillin $(1 \mu \mathrm{g})$, cotrimoxazole $(25 \mu \mathrm{g})$, penicillin (10U), cloxacillin $(10 \mu \mathrm{g})$, gentamicin $(10 \mu \mathrm{g})$, chloramphenicol $(30 \mu \mathrm{g})$, ciprofloxacin $(5 \mu \mathrm{g})$, and vancomycin $(10 \mu \mathrm{g})$. American Type Culture Collection (ATCC) 43300 was used as the control. The standard antimicrobial discs used for (HI Media) Pseudomonas aeruginosa were gentamicin $(10 \mu \mathrm{g})$, chloramphenicol $(30 \mu \mathrm{g})$, ciprofloxacin $(5 \mu \mathrm{g})$, ceftazidime $(30 \mu \mathrm{g})$, piperacillin $(100 \mu \mathrm{g})$, carbenecillin $(100 \mu \mathrm{g})$, and tobramycin $(10 \mu \mathrm{g})$. ATCC 27853 was used as control organism. 
R. K. Sanjana et al. Aerobic bacteriology of Chronic Suppurative Otitis Media.

\section{Results}

From 214 samples studied, microbiological culture was yielded from 192 samples (89.7\%). One hundred and sixty-eight samples (78.5\%) had a single organism isolated from the middle ear discharge, while the remaining 34 (15.9\%) had two or more organisms isolated. There were $12(05.6 \%)$ samples who had a sterile culture with no organism isolated. (Table-I)

\section{Table I}

Organism cultured from ear discharge Type of organism Total isolates Percentage

\begin{tabular}{lcc}
\hline Pure growth & 168 & $78.5 \%$ \\
Mixed growth & 34 & $15.9 \%$ \\
No growth & 12 & $05.6 \%$ \\
Total & $\mathbf{2 1 4}$ & $\mathbf{1 0 0 \%}$ \\
\hline
\end{tabular}

The most common causal organism isolated were Pseudomonas aeruginosa 59 (35.1\%) and Staphylococcus aureus 42 (25.0\%), of which 05 (03.0\%) were Methicillin Resistant Staphylococcus aureus (MRSA) followed by Coagulase negative staphylococcus 17 (10.1\%). Fungi accounted for $15(08.5 \%)$ of the total organisms isolated from the pure culture. (Table-II)

\section{Table II}

\section{Pure growth in culture of CSOM $(n=168)$}

Type of organism Total isolates Percentage Pseudomonas aeruginosa

\section{9}

35.1

Staphylococci aureus

$\begin{array}{lll}\text { (MSSA) } & 37 & 22.0 \\ \text { MRSA } & 05 & 03.0 \\ \text { CONS } & 17 & 10.1 \\ \text { Klebsiella species } & 13 & 07.7\end{array}$

$\begin{array}{lcc}\text { Escherichia coli } & 10 & 07.7 \\ \text { Proteus spps } & 05 & 03.0 \\ \text { Enterobacter spps } & 05 & 03.0 \\ \text { Citrobacter spps } & 02 & 01.1 \\ \text { Candida spps } & 06 & 03.5 \\ \text { Aspergillus spps } & 09 & 05.3 \\ \text { Total } & \mathbf{1 6 8} & \end{array}$

Pseudomonas species was isolated in 11 (32.3\%) of the total samples that yielded multi- organisms, Staph aureus in 07 (19.7\%), Klebsiella species in 04 (11.6\%), Proteus species 04 (11.6\%), followed by Escherichia coli 03 (08.8\%). (Table-III)

\section{Table III}

Mixed growth in culture of CSOM $(n=34)$

Type of organism No of isolates Percentage

\begin{tabular}{lll}
\hline Pseudomonas spps & 11 & 32.3
\end{tabular}

Staphylococci aureus

\begin{tabular}{lll} 
(MSSA) & 05 & 14.7 \\
MRSA & 02 & 05.8 \\
Klebsiella species & 04 & 11.7 \\
Proteus spps & 04 & 11.7 \\
Escherichia coli & 03 & 08.8 \\
Candida spps & 03 & 08.8 \\
Aspergillus spps & 02 & 05.8 \\
Total & $\mathbf{3 4}$ & \\
\hline
\end{tabular}

The antibiotic sensitivity pattern of the two most common isolates, Pseudomonas aeruginosa and Staphylococcus aureus (other than MRSA) is depicted in (Fig 1 \& 2) respectively. Among Pseudomonas aeruginosa, tobramycin has the highest susceptibility rate (93.2\%), followed by ceftazidime $(91.5 \%)$ and amikacin $(64.4 \%)$. 
Journal of College of Medical Sciences-Nepal,2011,Vol-7,No-2

Staphylococcus aureus is most susceptible to Cloxacillin (95.2\%), followed by erythromycin (83.3\%) and gentamicin (78.5\%).
All the seven isolates of MRSA were multidrug resistant; however, none of these were resistant to vancomycin.

\section{Antibiogram of Pseudomonas aeruginosa:}

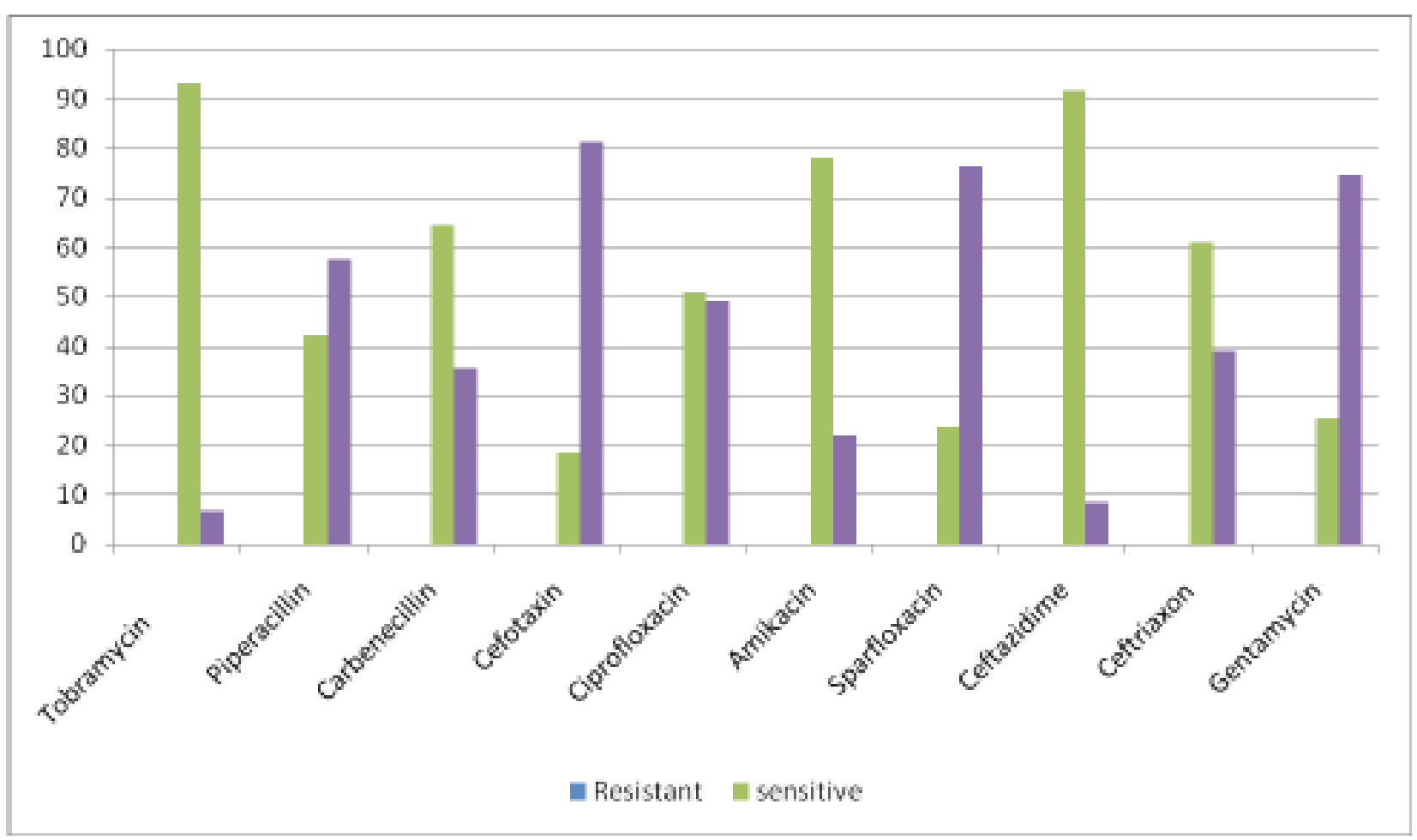

\section{Antibiogram of Staphylococcus aureus:}

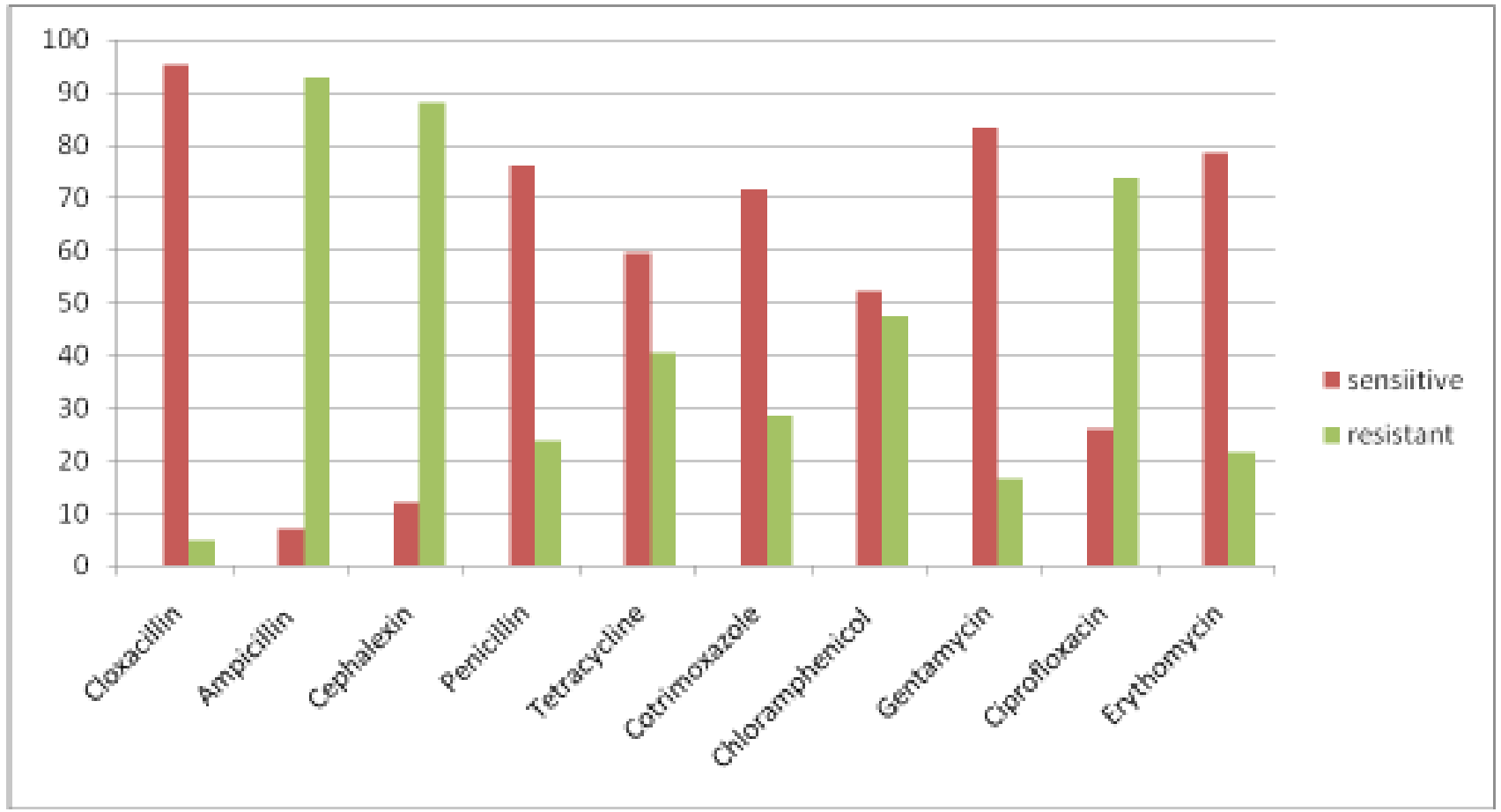


R. K. Sanjana et al. Aerobic bacteriology of Chronic Suppurative Otitis Media......

\section{Discussion}

CSOM is a condition of the middle ear that is characterised by persistent or recurrent discharge through a chronic perforation of the tympanic membrane. Due to the perforated tympanic membrane, bacteria can gain entry into the middle ear via the external ear canal. Infection of the middle ear mucosa subsequently results in ear discharge. It is a persistent disease with great risk of irreversible complications. Such complications range from persistent otorrhoea, mastoiditis, labyrinthitis, and facial nerve paralysis to more serious intracranial abscesses or thromboses. ${ }^{9}$ Though the incidence of such complications is low; they need to be borne in mind when faced by a patient with active CSOM. Early bacteriological diagnosis of all cases will assure accurate and appropriate effective therapy. Selection of antibiotic is influenced by its efficacy, resistance of bacteria, safety, risk of toxicity and costs. ${ }^{9}$

Knowledge of the local microorganisms, pattern and their antibiotic sensitivity is then essential to formulate a protocol for empirical antibiotic therapy.

Different studies on the microbiology of CSOM have revealed that the most frequently isolated bacteria were Pseudomonas aeruginosa, Staphylococcus aureus, Coagulase negative Staphylococcus, Proteus species, Klebsiella species and fungi. $5,9,10,11$

Our study has revealed that active infection of CSOM patients visiting the hospital is mainly due to Pseudomonas aeruginosa (35.1\%) and
Staphylococcus aureus (25.0\%)This finding is in tandem with the pattern of CSOM infection of the other studies mentioned above. There was also significantly high number of fungal isolates $(8.5 \%)$ in our study.

Review of literature about the studies done to find out bacterial flora in cases of CSOM in a state of India reveals that whereas Pseudomonas aeruginosa remained as the premier isolate in two studies, the isolation rate of Staphylococcus aureus in one of the study was quite low whereas other gram negative rods (i, e) Klebsiella pneumonia, Proteus mirabilis and Escherichia coli was relatively high. ${ }^{12,13}$ The third most commonly isolated organism, in our study, coagulase negative Staphylococcus (CONS) may represent skin flora contamination, and not be a true pathogen. However, one should not neglect any organism that is isolated from the infection.

Antimicrobial sensitivities of Pseudomonas aeruginosa in our study revealed that $93.2 \%$ isolates were sensitive to tobramycin while $91.5 \%$ of isolates were sensitive to ceftazidime and $77.9 \%$ to carbenecillin. On the other hand, $64.4 \%$ of isolates were sensitive to amikacin and $61.0 \%$ to ceftriaxone.50.8\% of the isolates were susceptible to ciprofloxacin.

A study carried out in Turkey ${ }^{14}$ in 1996 revealed only $6 \%$ of pseudomonas aeruginosa isolates to be resistant to ciprofloxacin; whereas, in South Korea in a study carried out in 2004, ciprofloxacin resistance was noted in $100 \%$ of isolates. ${ }^{15}$ In studies carried out in Pakistan revealed that more than 90\% 
isolates of Pseudomonas aeruginosa were sensitive to ciprofloxacin. $16,17,18,19$

In contrast, our study has shown only $50.8 \%$ of the isolates to be sensitive to ciprofloxacin. The declining sensitivity trend may be due to number of factors including injudicious use, inappropriate dosage, easy accessibility and developing enzymatic resistance of organisms especially Pseudomonas aeruginosa, Staphylococcus aureus and some Enterobacteriaceae against quinolones..$^{20,16}$

Similar activity is noted as far as in vitro activity of aminnoglycosides against $P$ seudomonas aeruginosa is concerned. Studies done in Singapore and India ${ }^{9}$ 13 had shown significantly higher percentage of Pseudomonas aeruginosa isolates sensitive to gentamicin compared to our studies where only $20.3 \%$ of the isolates were susceptible to this antibiotic. However, our finding is comparable to the study done by Ahmed Bel et, al..$^{17}$

As far as topical preparation is concerned for the treatment of CSOM, the otolaryngologist has either quinolones or aminoglycosides in their armory. Studies done by A H C Loy et, al; ${ }^{21}$ in 2002 published that gentamicin was most effective among topical ear drops. However, the authors discussed the controversies over the question of ototoxocity with the topical usage of aminoglycosides, such as gentamicin. In another study by Kardar AA et, al; ${ }^{22}$ had shown quinolones better results compared to topical aminoglycosides. Similar findings were also shown by other researchers, ${ }^{23,}{ }^{24}$ indicating the effectiveness of topical quinolones particularly against Pseudomonas aeruginosa and Staph aureus.
Hence, they would provide a viable alternative for the treatment of patients with active CSOM. At the same time, one should be concern about the fact that the widespread use of quinolones such as ofloxacin and ciprofloxacin could lead to emergence of resistance.

The antibiotic sensitivity pattern of Staphylococcus aureus (other than MRSA) in our study revealed that $95.2 \%$ of the isolates were sensitive to cloxacillin, $83.3 \%$ to gentamicin $78.5 \%$ to erythromycin and only $26.1 \%$ to ciprofloxacin. Least susceptibility was seen in ampicillin i.e. $07.1 \%$. The susceptibility pattern of Staph aureus found in our study against most of the antibiotics is almost consistent with other studies. ${ }^{16,17 \text { and } 18}$ However, our findings revealed lower sensitivity rate to ciprofloxacin $(26.1 \%)$ as compared to the study done by Ahmed B et al. ${ }^{17}$

\section{Conclusion}

Pseudomonas aeruginosa was the most common isolate followed by Staphylococcus aureus. Majority of Pseudomonas aeruginosa isolate (93.2\%) were sensitive to tobramycin and only $50.8 \%$ to ciprofloxacin. For Staphylococcus aureus (other than MRSA), $95.2 \%$ were sensitive to cloxacillin, $83.3 \%$ to gentamicin and only $26.1 \%$ of the isolates were sensitive to ciprofloxacin. Hence, our study revealed ciprofloxacin to be of less effective in the treatment of active CSOM. This report showed that in our hospital, tobramycin and cloxacillin may be included in the formulary to cover the most common isolates involved in CSOM. Nevertheless, more research needs to be done on the role of topical drugs in chronic suppurative otitis media. 


\section{References}

1. M. Goycoolea, L. Ruah. Definitions and Terminology. Otol Clin of North Am 1991; 24:757-61.

2. S. Couzos, T. Lea, R. Mullar et al. Effectiveness of ototopical antibiotics for CSOM in Aboriginal children, a community based multicenter double blind randomized controlled trial. Med J Aust 2003; 179(4): 185-90.

3. E. Alan, Dugdale. Management chronic suppurative otitis media. Med J Aus 2004; 180(2): 91-3.

4. Y. El Sayed. Bone conduction impairement in uncomplicated CSOM. Am J Otol. 1998; 19(1): 149-53.

5. N. Micro. Controlled multicenter studyon CSOM treated with topical application of ciprofloxacin $0.2 \%$ solution. Otolaryngol Head Neck Surg 2000; 123: 617-23.

6. R. Indudharan, J.A. Haq, S. Aigar. Antibiotics in CSOM. A bacteriologic study. Ann Otol. Rhino Laryngol 1999; 108 (5): 440-5.

7. J.P. Duiguild, J.G. Collee, A.G. Fraser. Laboratory strategy in the diagnosis of infective syndromes. In J.G. Collee, B.P. Marmion, A.G. Fraser, Simmons A. Mackie and Macartney practical medical microbiology. $14^{\text {th }}$ ed. London: 1996.

8. Clinlcal and Laboratory Instistute. Performance standards for antimicrobial suscerptyibility testing; $16^{\text {th }}$ information supplement (M100-S16).Clinical and Laboratory Standards Institute, Wayne, Pa: 2006.

9. A.H.C. Loy, A.L. Tan, P.K.S. Lu. Microbiology of Chronic Suppurative Otitis Media in Singapore. Singapore MedJ 2000; 43 (6): 296- 9.

10. O. Nekwa, Z.A. Shareef, A. Benayama. Anaerobes and fungi in chronic suppurative otitis media. Ann otorhino Laryngol 1997; 106(5): 649-52.

11. V. Khanna, J. Chander, N.M. Nagarkar et al. Clinicomicrobiologic evaluation of active tubotympanic type chronic suppurative otitis media. J Otol. 2000; 29(3): 148-53.

12. S.L. Hiremath, R.C. Kanta, M. Yeshwanth rao et al. Aerobic bacterial isolates of CSOM and their antibiotic sensitivity pattern. Ind Pract J 2001; 54(7): 486-9.

13. V.K. Poorey, A. Lyer. Study of bacterial flora in CSOM and its clinical significance. Ind J Otol. and Head and Neck Surg 2002; 54(2): 91-8.

14. C.H. Jang, S.Y. Park. Emergence of ciprofloxacin resistant pseudomonas in chronic suppurative otitis media. Cli Otol. 2004; 29(7): 321-3.

15. A. Altuntas, A. Aslam, A. Eren et al. Susceptibility of microorganisms isolated from chronic suppurative otitis media to ciprofloxacin . Eur Arch Otol-rhino-laryngol 1996; 253:364-6.

16. M.A. Aslam, Z. Ahmed, R. Azim. Microbiology and drug sensitivity patterns of chronic suppurative otitis media. J Col Physicians Surg Pak 2004; 8(14): 459-61.

17. B. Ahmed, S. Hydri, A.A.K. Afridi et al. Microbiology of ear discharge in Quetta. J Coll Physicians Surg Pak 2005; 15(9): 583-4.

18. Y. Taj, F. Essa, S.U. Kazmi. Pathological analysis of 596 cases of chronic suppurative otitis media in Karachi. J Coll Physicians Surg Pak. 2000; 10(4): 33-5.

19. A. Ahmed, J. Usman, R. Hashim. Isolates from chronic suppurative otitis media and their antibiotic sensitivity. Pak Armed Forces Med J 1999; 49(12): 82-5.

20. E. Vartiainen, J. Vartiainen. Effect of aerobic bacteriologyon the clinical presentation and treatment results of chronic suppurative media. $J$ Laryngol Otol 1996; 110: 315-8.

21. A.H.C. Loy, A. Tan, P.K.S. Lu. Microbiology of chronic suppurative otitis media in Singapore. Singapore Med J 2002; 43(6): 296-9. 
Journal of College of Medical Sciences-Nepal,2011, Vol-7,No-2

22. A.A. Kardar, M. Usman, S. Tirmizi. Topical quinolone versus topical aminoglycosides in the medical management of chronic suppurative otitis media; A comparative trial. J Surg Pak 2003; 8 (4): 6-9.

23. A.P. Yuen, P.Y. Chau, Wei. Bacteriology of chronic suppurative otitis media; ofloxacin susceptibility. J Otol. 1995; 24(3): 206-8.
24. A.S. Agro, E.T. Garner, J.W. Wright et al. Clinical trial of ototopical ofloxacin for treatment of chronic suppurative otitis media. Cln Ther 1998; 20 (4): 744-59. 